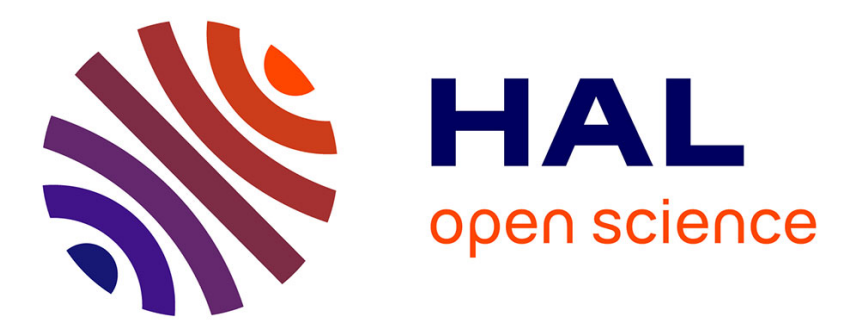

\title{
On the intermittency of gravity wave momentum flux in the stratosphere
}

\author{
Albert Hertzog, J.M. Alexander, Riwal Plougonven
}

\section{To cite this version:}

Albert Hertzog, J.M. Alexander, Riwal Plougonven. On the intermittency of gravity wave momentum flux in the stratosphere. Journal of the Atmospheric Sciences, 2012, 69 (11), pp.3433-3448. 10.1175/JAS-D-12-09.1 . hal-01113617

\section{HAL Id: hal-01113617 https://hal.science/hal-01113617}

Submitted on 7 Feb 2015

HAL is a multi-disciplinary open access archive for the deposit and dissemination of scientific research documents, whether they are published or not. The documents may come from teaching and research institutions in France or abroad, or from public or private research centers.
L'archive ouverte pluridisciplinaire HAL, est destinée au dépôt et à la diffusion de documents scientifiques de niveau recherche, publiés ou non, émanant des établissements d'enseignement et de recherche français ou étrangers, des laboratoires publics ou privés. 


\title{
On the Intermittency of Gravity Wave Momentum Flux in the Stratosphere
}

\author{
Albert HeRTZOG \\ Laboratoire de Météorologie Dynamique, École Polytechnique, IPSL, Palaiseau, France \\ M. JOAN ALEXANDER \\ NorthWest Research Associates, CoRA Office, Boulder, Colorado \\ RiWAL PlougonVEN \\ Laboratoire de Météorologie Dynamique, École Normale Supérieure, IPSL, Paris, France
}

(Manuscript received 6 January 2012, in final form 12 June 2012)

\begin{abstract}
In this article, long-duration balloon and spaceborne observations, and mesoscale numerical simulations are used to study the intermittency of gravity waves in the lower stratosphere above Antarctica and the Southern Ocean; namely, the characteristics of the gravity wave momentum-flux probability density functions (pdfs) obtained with these three datasets are described. The pdfs consistently exhibit long tails associated with the occurrence of rare and large-amplitude events. The pdf tails are even longer above mountains than above oceanic areas, which is in agreement with previous studies of gravity wave intermittency in this region. It is moreover found that these rare, large-amplitude events represent the main contribution to the total momentum flux during the winter regime of the stratospheric circulation. In contrast, the wave intermittency significantly decreases when stratospheric easterlies develop in late spring and summer. It is also shown that, except above mountainous areas in winter, the momentum-flux pdfs tend to behave like lognormal distributions. Monte Carlo simulations are undertaken to examine the role played by critical levels in influencing the shape of momentum-flux pdfs. In particular, the study finds that the lognormal shape may result from the propagation of a wave spectrum into a varying background wind field that generates the occurrence of frequent critical levels.
\end{abstract}

\section{Introduction}

Mesoscale gravity waves transport energy and momentum from the lower layers of the atmosphere to the stratosphere and mesosphere (e.g., Holton et al. 1995). As a result of the atmospheric decrease of density with altitude, the conservation of energy and momentum implies an increase of gravity wave amplitudes as the waves propagate upward. Gravity waves eventually break when their associated disturbances become too large and trigger instabilities. Such instabilities can also be generated when the background wind refracts the waves toward high vertical wavenumbers (or, equivalently, to intrinsic frequencies close to the inertial frequency), a process

Corresponding author address: Albert Hertzog, Laboratoire de Météorologie Dynamique, École Polytechnique, 91128 Palaiseau CEDEX, France.

E-mail: albert.hertzog@lmd.polytechnique.fr known as critical-level filtering. At those levels, the wave momentum is transferred to the mean flow and acts as a forcing to the general circulation. Through these processes, the extratropical gravity waves significantly contribute to the generation of the meridional, global-scale Brewer-Dobson circulation, which is responsible for driving the middle atmosphere out of radiative equilibrium (e.g., Holton 1983; Andrews et al. 1987). To incorporate these effects and to simulate a realistic stratosphere and mesosphere, most atmospheric general circulation models (GCMs) have to use dedicated gravity wave drag (GWD) parameterizations, as the model resolutions are still currently too coarse to explicitly resolve the whole spectrum of gravity waves (e.g., Morgenstern et al. 2010).

A wide body of studies has described the atmospheric gravity wave field in terms of "universal spectra" (e.g., VanZandt 1982; Smith et al. 1987; Fritts et al. 1988; Sidi et al. 1988; Fritts and Lu 1993). Various theories have been proposed to explain the observed spectral slopes, 
in particular (but not only), the $\mathrm{m}^{-3}$ scaling of the gravity wave energy spectrum with vertical wavenumbers (Dewan and Good 1986; VanZandt and Fritts 1989; Weinstock 1990; Hines 1991; Medvedev and Klaassen 1995; Dewan 1997; Souprayen et al. 2001); and nonorographic gravity wave drag parameterizations have been developed by implementing those spectral approaches (Hines 1997a,b; Warner and McIntyre 2001; Scinocca 2003).

In contrast, many observational studies have highlighted that gravity waves are generally observed as wave packets rather than as a spectral continuum (e.g., Pfister et al. 1993; Alexander and Pfister 1995; Eckermann and Preusse 1999; Plougonven et al. 2008). Instantaneous snapshots of high-resolution numerical simulations also tend to exhibit wave signatures that can be associated with wave packets (Watanabe et al. 2008). In this spirit, Lindzen (1981) and Alexander and Dunkerton (1999) have developed gravity wave drag schemes that compute the momentum deposition induced by a collection of individual wave packets. This packet-like description implicitly stresses that gravity wave activity in the atmosphere is (at least to some extent) intermittent; that is, significant variations in the amplitude of wave-induced disturbances can occur over time scales comparable to those of the wave packets.

The wave intermittency essentially results from two factors: 1) The processes leading to gravity wave generation can themselves be intermittent. For a given mountain shape, for instance, the amplitude of the generated orographic waves strongly depends on the wind speed and direction near the mountain top (e.g., Smith 1979, and reference therein). 2) The wave propagation in the atmosphere, and in particular the refraction (e.g., the focusing in the jet core) and filtering imposed by the background wind, can furthermore modulate the wave activity. Bühler (2003) has shown that taking into account the source intermittency in gravity wave drag parameterizations can produce significantly different results than those induced by (generally assumed) stationary sources. In particular, the altitude level where the waves break is arguably one of the parameterization outputs that is the most sensitive to wave amplitudes.

In this study, the gravity wave intermittency is analyzed by using momentum fluxes provided by stratospheric long-duration balloons (Hertzog et al. 2008), by the spaceborne infrared High Resolution Dynamics Limb Sounder (HIRDLS) instrument (Alexander et al. 2008), as well as by high-resolution numerical simulations performed with the nonhydrostatic Weather Research and Forecasting Model (WRF) (Plougonven et al. 2010). The analysis will be focused on the Vorcore balloon campaign, which took place over Antarctica and the surrounding ocean between September 2005 and February 2006 (Hertzog et al. 2007). A brief overview of the different datasets will be given in the next section. The intermittency of gravity wave activity will then be described by directly looking at the shape of the momentum-flux probability density functions (pdfs). Recent studies have shown that pdfs of gravity wave potential energy (Baumgaertner and McDonald 2007) and momentum flux (Alexander et al. 2010) in the lower stratosphere exhibit broad tails that are associated with the occurrence of rare but intense gravity wave events. The aim of this article is to further analyze the momentumflux pdfs over Antarctica and the surrounding ocean, and in particular to describe its variations with geographical location, height, and season. Section 3 will be devoted to these observational and numerical results. In section 4 , we will show that the wave filtering by the background wind can be responsible for producing some characteristics of the pdf shapes that are commonly observed in the atmosphere. The last section of the article will provide some concluding remarks.

\section{Datasets}

\section{a. Long-duration balloons}

The balloon dataset used in this study has been gathered during the flights of 27 superpressure balloons performed in the frame of the Vorcore campaign in Antarctica (Hertzog et al. 2007). During the campaign, 8.5- and $10-\mathrm{m}$ diameter balloons that typically drift around $17 \mathrm{~km}(75 \mathrm{hPa})$ and $19 \mathrm{~km}(55 \mathrm{hPa})$, respectively, were used. Such balloons can fly for several months on constant-density (isopycnal) surfaces in the atmosphere. They are advected by the wind and therefore behave as quasi-Lagrangian tracers in the stratosphere. The balloon flights took place between early September 2005 and early February 2006, with a maximum of balloons flying simultaneously in October and November. The Vorcore dataset is thus primarily representative of the lower polar stratosphere during austral spring. In particular, all balloons were launched inside the stratospheric polar vortex, and most of them drifted in the stratospheric jet, close to the vortex edge until the vortex broke in mid-December.

During the campaign, each balloon was equipped with a meteorological instrument monitoring the air temperature and pressure every $15 \mathrm{~min}$ along the flight, as well as with a GPS receiver providing the balloon position at the same sampling rate. The zonal and meridional components of the wind were deduced from the horizontal GPS positions by finite differences, assuming that the balloons perfectly follow the horizontal wind (e.g., Vial et al. 2001). Further details on the instruments, on 
the balloon flights, and on the Vorcore campaign in general can be found in Hertzog et al. (2007).

The vertical fluxes of zonal, meridional, and total horizontal gravity wave momentum are estimated from the observations by computing the correlation between horizontal and vertical velocity disturbances induced by gravity waves (Hertzog and Vial 2001; Boccara et al. 2008). While the horizontal velocity disturbances are directly measured, the vertical ones are deduced from the vertical displacement of the isopycnal surface on which the balloons are flying. Separating the gravity wave component from the planetary wave component in the observed disturbances is an easy task in longduration balloon observations, at least at high latitudes, because these measurements are done in the intrinsic frame of reference (moving with the background wind) in which there exists a clear spectral gap between both kind of motions (e.g., Hertzog et al. 2002b).

The same momentum-flux dataset as the one used in Vincent et al. (2007) and Hertzog et al. (2008) is used in this study: in particular, as a result of the sampling frequency of Vorcore observations, only waves with intrinsic periods longer than $1 \mathrm{~h}$ are considered. For each flight, a value of gravity wave momentum flux is obtained every $15 \mathrm{~min}$. This is achieved by doing a wavelet analysis of the observed time series, and thus decomposing the disturbances in the time-intrinsic frequency $(t, \hat{\omega})$ space. The correlations between velocities are computed in this space, which allows us to estimate the direction of propagation of individual wave packets, and the associated amplitudes in zonal, meridional, and vertical velocities $u^{\prime}, v^{\prime}$, and $w^{\prime}$, respectively, as well as in the velocity along the wave direction of propagation $u_{\|}^{\prime}$. At each observation time, the momentum fluxes are then summed in the $\hat{\omega}$ direction over the wave packets, so that time series of total, zonal, and meridional momentum fluxes are obtained, that is,

$$
\begin{aligned}
& \rho \overline{u_{\|}^{\prime} w^{\prime}}(t) \equiv \rho \sqrt{\overline{u^{\prime} w^{\prime}}(t)+\overline{v^{\prime} w^{\prime}}{ }^{2}(t)}, \quad \rho \overline{u^{\prime} w^{\prime}}(t), \quad \text { and } \\
& \rho \overline{v^{\prime} w^{\prime}}(t)
\end{aligned}
$$

respectively, where $\rho$ is the atmospheric density at the balloon flight level and the overbar denotes the average over wave packets. These time series are then used to compute the pdfs discussed in the section 3 .

\section{b. HIRDLS}

HIRDLS is an infrared limb-scanning instrument on the Aura satellite. The satellite flies in a high inclination orbit in the A-Train satellite constellation. HIRDLS views the limb at a fixed $47^{\circ}$ angle from the orbit track, which gives a north/south asymmetry in the measurement

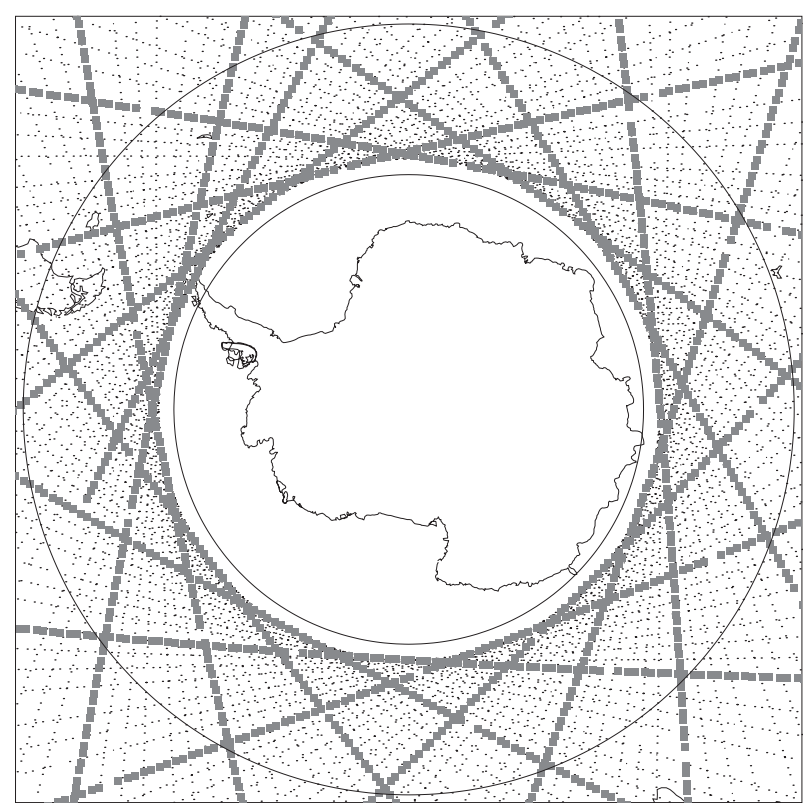

FIG. 1. Locations of HIRDLS profiles over the South Pole during one week (black dots) of measurements in October and for a single day (thick gray dashes). Circles mark latitudes $65^{\circ}$ and $50^{\circ} \mathrm{S}$. This latitude band is used for the comparison between HIRDLS and long-duration balloon momentum fluxes.

track [see Gille et al. (2008) for further description of the instrument, measurements, and noise characteristics]. Each vertical scan is completed in about $8 \mathrm{~s}$, giving an approximately constant horizontal spacing between vertical profiles of about $100 \mathrm{~km}$. The minimum southern latitude covered is approximately $63.5^{\circ} \mathrm{S}$, and at this extreme latitude, the HIRDLS measurement track is longitudinal (see Fig. 1), while in the equatorial region it is almost latitudinal. For this study we use only HIRDLS measurements south of $50^{\circ} \mathrm{S}$.

To estimate the momentum flux from HIRDLS temperature measurements, the procedure described in Alexander et al. (2008) is applied. Briefly, each pair of adjacent profiles is analyzed with the $S$ transform in the vertical, and a height-dependent vertical wavenumber $m$ covariance spectrum is computed. The maximum in the covariance spectrum at each altitude determines the dominant vertical wavenumber signal present in both profiles, and the difference in phase in this dominant signal gives an estimate of the horizontal wavenumber $k$. The momentum flux is proportional to the temperature covariance times the ratio $m / k$. It is recalled here that $k$ is the apparent horizontal wavenumber along the line joining two profiles, and it will generally be smaller than the true wavenumber along the line perpendicular to wave phase fronts. Note also that a minimum vertical wavenumber must be chosen, and here it is set to $(24 \mathrm{~km})^{-1}$. 
Prior to the gravity wave analysis, a "background" temperature is removed from the HIRDLS temperature profiles. The background temperature is defined by a zonal wavenumber (wn) 0-5 signal derived from S-transform analysis of the data as a function of longitude collected in $2.5^{\circ}$ latitude bins. Tests were run with maximum wavenumbers $3-6$, and a value of 5 was found to give the best description of sharp gradients in distorted vortex cases without introducing artificial oscillations surrounding poorly resolved features near wn $=6$. Note that the use of the $S$ transform with wn $=1-5$ gives a better description of the longitudinal variations than a traditional Fourier analysis with the same wavenumber range because it allows for the localization of the signals in longitude as with a wavelet analysis.

\section{c. WRF simulations}

The numerical mesoscale simulations were carried out with WRF (Skamarock et al. 2008), following a configuration determined by a preliminary sensitivity study (Plougonven et al. 2010). The resulting gravity wave field in the lower stratosphere is analyzed in Plougonven et al. (2012). The domain is $10000 \mathrm{~km} \times 10000 \mathrm{~km}$, with a horizontal resolution of $20 \mathrm{~km}$, and uses a Lambert conformal projection. The grid has 120 levels in the vertical and extends to about $5 \mathrm{hPa}$, that is, about $36 \mathrm{~km}$. The level spacing in the vertical is kept close to constant at $300 \mathrm{~m}$. Parameterization of microphysics uses the WRF Single-Moment 5-Class Microphysics Scheme, and the Noah land surface model is used for land surface, as recent modifications have been added for processes over ice and snow in the recent WRF version 3 .

To cover 2 months of the Vorcore campaign, a succession of 29 short runs were carried out. Each run lasted 3 days, the first day being for spinup and the last 2 days for analysis. There is an overlap of 1 day between successive runs. The first day started at 0000 universal time (UT) 20 October 2005 and the last day ended at 0000 UT 18 December 2005. Stitched together, these runs provide 58 simulated days for analysis, from 21 October to 18 December. In this study, we will only use the November runs to analyze the momentumflux distribution.

For each simulation, the initial condition and the boundary conditions are prepared from the analyses provided by the European Centre for Medium-Range Weather Forecasts (ECMWF) operational Integrated Forecast System. The short length of each run and the predictability of the flow at these latitudes guarantees that the simulations stay relatively close to the analyses, without having to use data assimilation or nudging, which could have contaminated our analysis with spurious gravity waves.

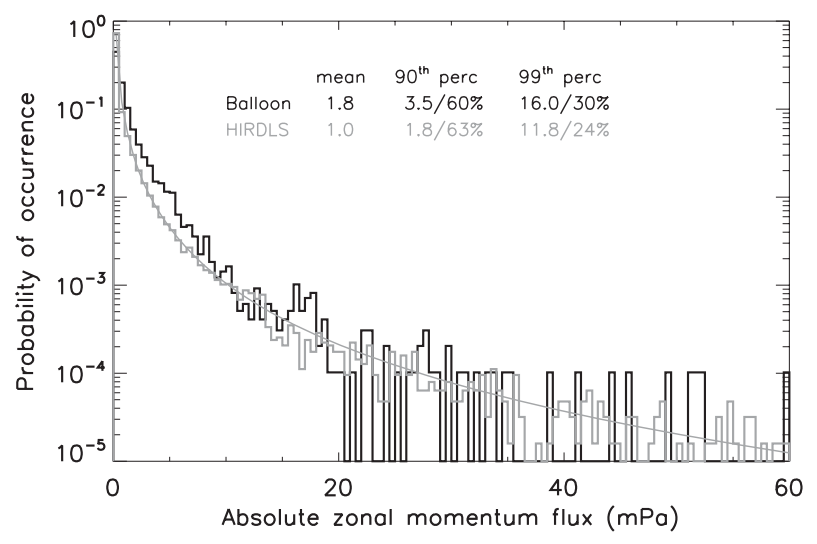

FIG. 2. Pdf (histogram style) of absolute zonal momentum fluxes obtained with balloon (black) and HIRDLS (gray) observations at $20 \mathrm{~km}$ between $50^{\circ}$ and $65^{\circ} \mathrm{S}$ in October 2005. The continuous gray line shows the pdf of a lognormal distribution with the same geometric mean and standard deviation as the HIRDLS distribution. For each distribution, the (arithmetic) mean, and 90th and 99th percentiles are displayed (all values in $\mathrm{mPa}$ ). The percentage of total flux associated with fluxes larger than the percentiles are furthermore indicated: for instance, in the balloon dataset, fluxes larger than $3.5 \mathrm{mPa}$ occur $10 \%$ of the time (90th percentile) but correspond to $60 \%$ of the total absolute zonal momentum flux.

As described in Plougonven et al. (2012), the zonal and meridional momentum fluxes were calculated in the following way: in the three components of the wind field (along $x, y$, and $z$ in the model grid), the small-scale components $\left(u_{x}^{\prime}, v_{y}^{\prime}\right.$, and $\left.w^{\prime}\right)$ were identified using a moving filter with a Hamming window of width $1000 \mathrm{~km}$. The horizontal wind fluctuations were converted to zonal and meridional components $u^{\prime}$ and $v^{\prime}$. Total, zonal, and meridional momentum fluxes were then obtained as $\rho \sqrt{\left(u^{\prime} w^{\prime}\right)^{2}+\left(v^{\prime} w^{\prime}\right)^{2}}, \rho u^{\prime} w^{\prime}$, and $\rho v^{\prime} w^{\prime}$, respectively. The resulting field was smoothed using a moving window of radius $100 \mathrm{~km}$, so as to be comparable with the resolution of satellite data. It was checked that the results were not very sensitive to this final filtering.

\section{Probability density functions}

\section{a. Overall pdfs}

Figure 2 displays the pdf of absolute zonal momentum fluxes $\rho\left|\overline{u^{\prime} w^{\prime}}\right|$ obtained with balloon observations, together with the absolute momentum-flux pdf obtained with HIRDLS at $20 \mathrm{~km}$. The pdfs have been constructed with all the (balloon and HIRDLS) measurements performed in October 2005 in the $50^{\circ}-65^{\circ} \mathrm{S}$ latitude band, where both datasets overlap. As illustrated in Fig. 1, these latitudes primarily correspond to the ocean surrounding Antarctica. In contrast, orographic areas, such as the northern extremity of the Antarctic Peninsula, the southern tip of South America, as well as a number of 
islands (South Georgia, Heard, Kerguelen, etc.) that are expected to generate significant gravity wave activity (e.g., Eckermann and Preusse 1999; Wu 2004; Alexander and Teitelbaum 2007; Plougonven et al. 2008; Alexander et al. 2009), only represent a marginal fraction of this whole surface.

The Vorcore balloon and HIRDLS observations produce gravity wave momentum-flux distributions that agree fairly well. The relatively small differences between the balloon and HIRDLS pdfs likely result from the very different sampling of the atmosphere by both techniques (in particular, the greater number of HIRDLS observations explains its better representation of the most unlikely momentum-flux events), the details in which momentum fluxes are computed in both datasets, as well as from the small vertical distance between the balloon and HIRDLS observations (between 1 and $3 \mathrm{~km}$ ).

Most importantly, both balloon and HIRDLS pdfs are positively skewed. Waves with small fluxes are thus by and large the most likely events: the probability of observing fluxes less than a few millipascals is, for instance, equal or greater than $90 \%$ in both datasets (see percentiles in Fig. 2). In contrast, both pdfs exhibit a broad tail of rare events, which extends toward large momentum fluxes. Following Lorenz (1905), such property can be further assessed by looking at the percentage of the total flux corresponding to fluxes larger than a given quantile as follows:

$$
\frac{\sum_{f^{i}>f_{q}} f^{i}}{\sum_{i=1}^{N} f^{i}} \times 100
$$

where $N$ is the total number of observations, $f^{i}$ is the $i$ th flux observation, and $f_{q}$, the $q$ th quantile, verifies:

$$
\frac{\sum_{f^{i}>f_{q}} i}{N}=1-q .
$$

These percentages are displayed in Fig. 2 for the 90th and 99th quantiles. In both datasets, it is found that about $60 \%$ of the total flux is due to only the $10 \%$ largest wave events, while the $1 \%$ largest events still explain about $25 \%$ or more of the total flux. Sporadic wave packets carrying a few hundreds of millipascals (not shown in Fig. 2), that is, about 100 times the mean flux, actually appear in the balloon and HIRDLS observations, and explain the previous statistics.

In summary, such kinds of pdfs, and in particular the observed presence of long tails, are readily responsible for the intermittent character of gravity wave activity in the stratosphere. These two features were already reported in Alexander et al. (2010) (cf. their Fig. 6), who compared the same datasets for the whole Vorcore period. The agreement between both distributions is nevertheless better in Fig. 2 because first, the balloon and HIRDLS sampling are very similar over the Southern Ocean in October; and second, the balloon absolute zonal momentum-flux pdf is compared to the HIRDLS pdf here instead of the total momentum flux in Alexander et al. (2010). The resemblance of the pdfs also likely results from the fact that the subrange of the whole gravity wave field that can be observed by Vorcore balloons and HIRDLS is very similar, although the definition of the observational filter differs for each observing technique (long intrinsic frequency for the balloons, primarily long horizontal wavelength for HIRDLS) (Alexander et al. 2010, their Fig. 8). This agreement finally suggests that the skewed pdfs reported in Fig. 2 likely constitute a real feature of gravity waves in the stratosphere.

Figure 2 also displays a theoretical lognormal distribution $\Lambda(\mu, \sigma)$, which has the same geometric mean $e^{\mu}$ and standard deviation $e^{\sigma}$ than those estimated on the HIRDLS momentum-flux observations ( $\mu$ and $\sigma$ are also the arithmetic mean and standard deviation of $\ln \Lambda$, respectively, which is a normal random variate by definition). This lognormal pdf nicely fits the HIRDLS distribution. A lognormal fit of the balloon pdf (not shown in the figure) produces the same results. Nastrom and Gage (1985) first reported the lognormal behavior of disturbance variances at mesoscales in the atmosphere, and more recently Baumgaertner and McDonald (2007) succeeded in fitting the occurrence of gravity wave potential energies over Antarctica by a lognormal distribution. We shall come back to the lognormal behavior of the observed pdfs later in the article.

\section{b. Pdfs over mountains and smooth terrains}

As previously mentioned, the $50^{\circ}-65^{\circ} \mathrm{S}$ latitude band essentially includes oceanic areas. Hertzog et al. (2008) used a couple of proxies to show that the intermittency of gravity waves could be significantly larger over mountains. It is thus worthwhile to look how this different behavior is reflected in the momentum-flux pdf. Figure 3 displays the balloon pdfs for the whole Vorcore period over mountainous and nonmountainous areas. The geographical criterion used to distinguish between the two areas is the same as the one used by Hertzog et al. (2008), and the nonmountainous areas typically correspond to the Southern Ocean and the Antarctic Plateau. The most obvious difference between the two pdfs is the presence of a much longer tail in the gravity wave momentum-flux distribution over mountains. Consequently, larger fluxes do occur more frequently 


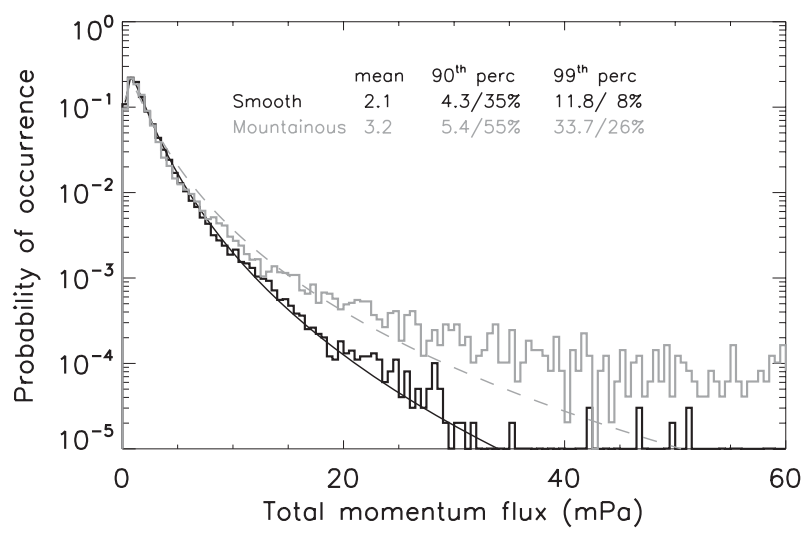

FIG. 3. Pdfs (histogram style) of total momentum fluxes obtained with long-duration balloons during the whole Vorcore period. Pdf over oceanic or continental flat areas (black), pdf over mountainous areas (gray) (see text for details). The continuous black (dashed gray) line shows the theoretical pdf of a lognormal distribution with the same geometric mean and standard deviation as the observed oceanic (mountainous) pdf.

over mountainous terrains than above smoother areas as already reported in Nastrom and Fritts (1992) for instance, and shown here by the 99th percentiles of the distributions. But the different pdf shapes also mean that the largest (and rarest) fluxes do not have the same contribution to the total flux over both kinds of terrain: as reported in Fig. 3, the 1\% highest fluxes explain 26\% of the total flux over mountains (for the whole campaign) versus $8 \%$ over smoother areas. These distributions are thus consistent with the greater intermittency over mountains as mentioned above; Fig. 3 also shows that the mountainous and nonmountainous pdfs look very similar for small momentum fluxes (up to about $10 \mathrm{mPa}$ ). This tends to suggest that there exists a background gravity wave activity that can be observed virtually everywhere in the stratosphere, and that it may therefore justify the use of globally homogeneous nonorographic gravity wave drag schemes in GCMs. Note, however, that the present results only apply to the southernmost latitudes of the globe and need further confirmation at global scale.

In agreement with the HIRDLS pdf in October over the ocean, Fig. 3 also illustrates that the nonmountainous pdf is well fitted by a lognormal distribution, even for momentum fluxes greater than $20 \mathrm{mPa}$ that are marginally likely. In contrast, the lognormal distribution is obviously not an accurate model over mountains: it slightly overestimates the observed occurrence of small momentum fluxes, and it strongly underestimates the observed occurrence of the largest ones.

Figure 4 shows the pdfs of total momentum flux obtained from the high-resolution WRF simulations during November 2005 at $17 \mathrm{~km}$ over the same smooth and

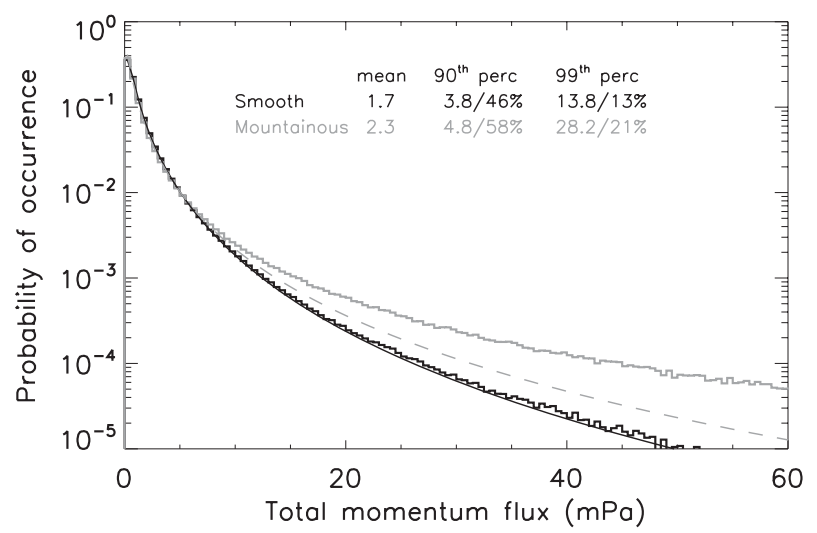

FIG. 4. Pdfs (histogram style) of total momentum fluxes obtained with the WRF simulations at $17 \mathrm{~km}$ for November over the same mountainous (black) and smooth (gray) areas as those displayed in Fig. 3. As in Fig. 3, the continuous lines show the corresponding lognormal pdfs.

mountainous regions than those shown for the balloon dataset. It may be first noted that the gravity wave momentum fluxes obtained with the WRF simulations compare very well with those obtained with the balloon [as reported in Plougonven et al. (2010)] and HIRDLS observations, even though we made no effort to adjust the observational filter of each dataset. The numerical simulations furthermore confirm the behavior deduced from the balloon observations, that is, 1) the pdf over mountainous terrains has a much broader tail than the one over smoother regions; 2) the smooth pdf agrees well with the lognormal distribution; 3 ) the lognormal distribution underestimates the largest events over mountains; and 4) both pdfs are very similar at the low momentum-flux values.

A few differences with the pdfs shown in Figs. 3 and 4 should, however, be noted. First, the mean momentum values are slightly weaker in the WRF simulations than in the balloon dataset. This difference is likely caused by the time period associated with each pdf: while the WRF distributions correspond to November, the balloon pdfs cover the whole period from September to January, with a primary emphasis for October and November (i.e., somewhat prior in the year). We will come back to seasonal effects on the pdf shape in the next section. The other difference is in the contribution of the largestamplitude waves over smooth terrains to the total momentum flux, which is somewhat larger in the WRF simulations than in the balloon observations. The relatively weak coverage of the Southern Ocean by the balloons (especially with respect to its "perfect" counterpart in the simulations) may readily explain why the most infrequent events are better sampled in the WRF simulations (Plougonven et al. 2012). 

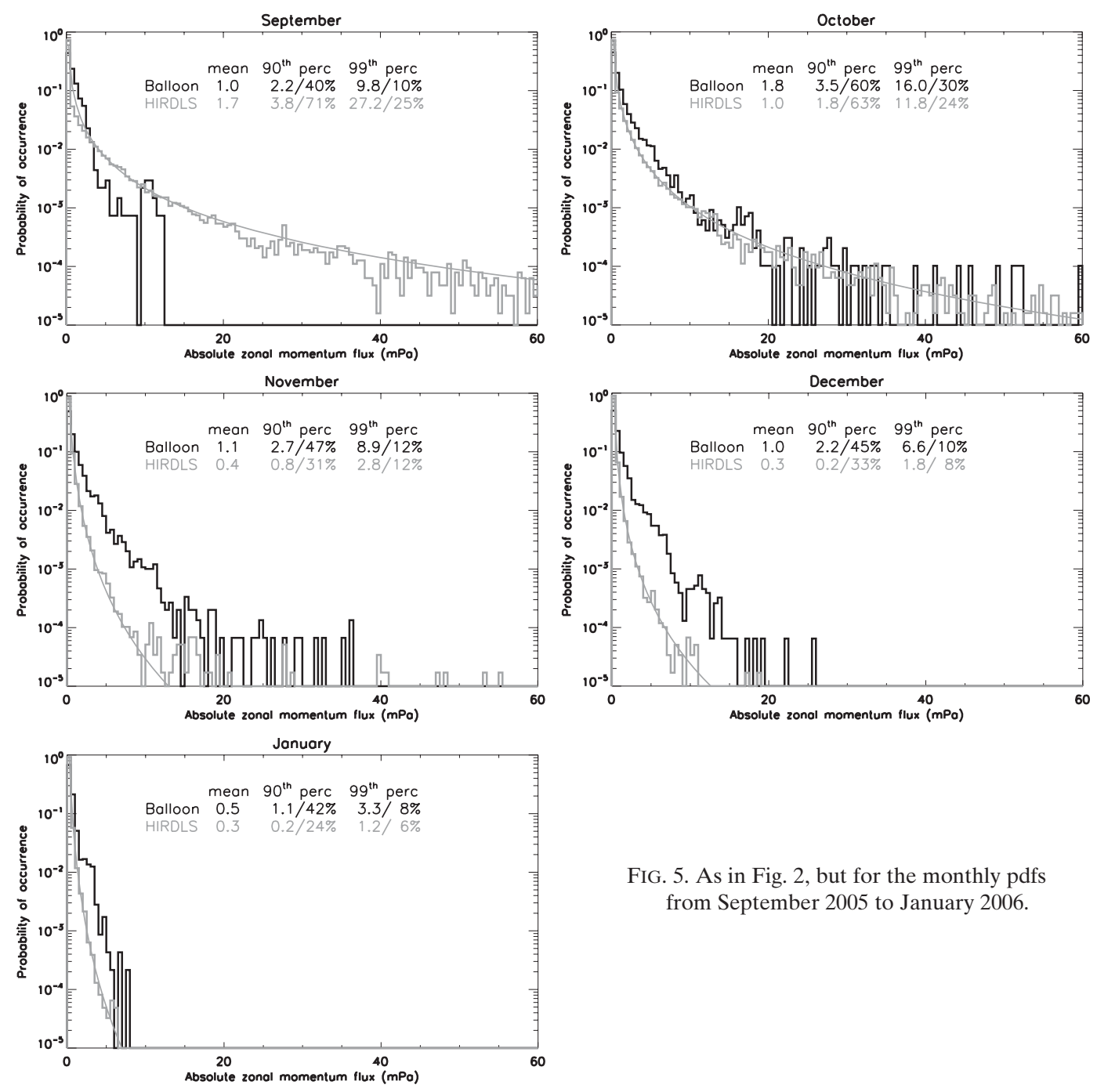

FIG. 5. As in Fig. 2, but for the monthly pdfs from September 2005 to January 2006.

\section{c. Seasonal evolution}

The evolution from September 2005 to January 2006 of the balloon and HIRDLS absolute zonal momentumflux pdf is shown in Fig. 5. As mentioned previously, the pdfs have been computed with observations within the $50^{\circ}-65^{\circ} \mathrm{S}$ latitude band, where both datasets overlap. Most of the differences between the HIRDLS and balloon pdfs can be attributed to a different sampling of this area. In September, the balloons are essentially drifting at more southern latitudes, and the corresponding pdf likely lacks enough observations for being representative of subpolar latitudes, especially for the infrequent events; in November and December, HIRLDS samples this area irrespective of the position of the disturbed vortex, whereas a large majority of the balloons are still drifting in the vortex (and even close to its edge). The magnitude of gravity wave filtering by the background

wind (strong and eastward in the vortex, lighter and sometimes westward outside the vortex) experienced in each dataset during those months is therefore quite different. In January, finally, the vortex has disappeared, but the zero wind surface is located close to $20 \mathrm{~km}$ and could therefore explain the relatively lower momentum fluxes observed by HIRDLS as compared to the balloons.

Despite these differences, both pdfs exhibit the same and robust evolution: from late winter to early summer, the tail of large values progressively disappears. This is further illustrated by the decrease of the monthly-mean momentum flux in each dataset (with the exception of the September distribution in the balloon dataset, for the reasons mentioned above), and by the concurrent decrease in the contribution to the total flux of the $10 \%$ and $1 \%$ rarest fluxes. In other words, the lowerstratosphere gravity wave activity not only diminishes 


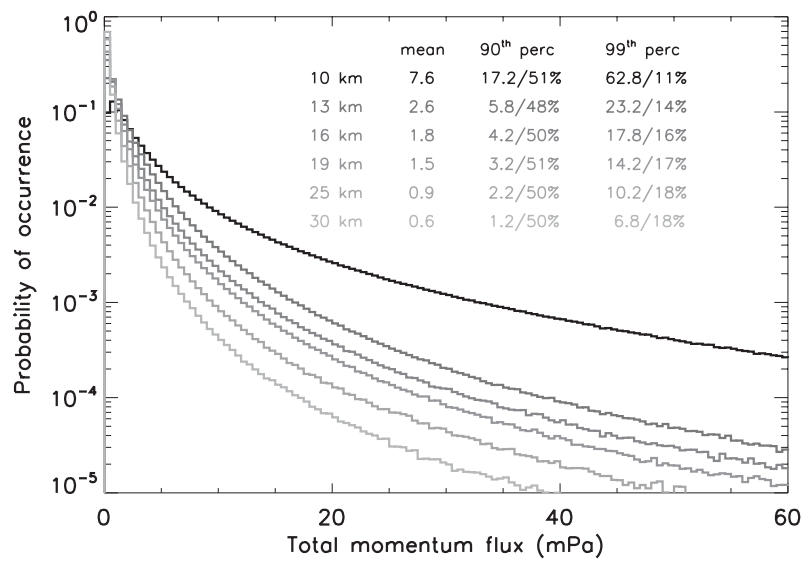

FIG. 6. Pdfs of total momentum fluxes obtained with the WRF simulations at various heights for November. The pdfs are representative of the whole simulation domain.

during this period over the Southern Ocean but also becomes less and less intermittent. These two aspects are likely caused by the progressive reversal of the zonal wind that inhibits the upward propagation of a large portion of the gravity wave spectrum.

\section{d. Pdf evolution with height}

Figure 6 displays the total momentum-flux pdfs in WRF simulations at various heights over the whole simulation domain. Although the WRF domain was centered over the South Pole, it encompasses subpolar latitudes so that the lowest altitudes shown are likely partly located in the troposphere. These pdfs may thus correspond to momentum fluxes that are not only associated with gravity wave disturbances but also tropospheric processes (e.g., upper-level fronts).

With this reservation, the behavior of momentum-flux pdfs with height is notably systematic in the WRF simulations. As one moves away from the tropospheric gravity wave sources, the momentum fluxes regularly decrease due to the progressive dissipation of the wave field. But even more impressively, the pdf evolution suggests self-similarity: the 90th and 99th percentiles almost steadily correspond to 2 and 10 times the distribution mean. Similarly, the contribution of the $10 \%$ and $1 \%$ rarest momentum fluxes amounts to about $50 \%$ and $15 \%-20 \%$, respectively, of the total flux whatever the altitude. In contrast with the seasonal evolution of the pdfs, the November WRF simulations tend therefore to show that the intermittency of the gravity wave momentum fluxes keeps constant with altitude (at least up to the middle stratosphere).

Figure 7 displays the absolute momentum-flux pdfs obtained with HIRDLS measurements at 20,30, and $40 \mathrm{~km}$. As HIRDLS observations do not cover latitudes

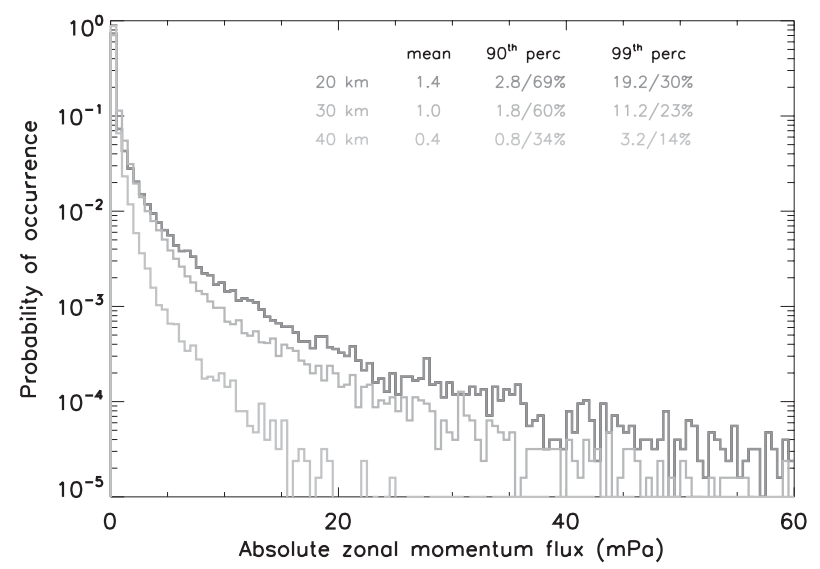

FIG. 7. Pdfs of absolute zonal momentum fluxes obtained with HIRDLS observations at various heights between $50^{\circ}$ and $65^{\circ} \mathrm{S}$ in September and October 2005.

poleward of $65^{\circ} \mathrm{S}$, we have chosen for a better comparison with the previous figure to show the HIRLDS pdfs for September and October, which correspond to an eastward circulation in the stratosphere at these subpolar latitudes as in November over the South Pole. The magnitude and general height evolution of HIRDLS pdfs compare very well with the WRF ones. Furthermore, as previously noted, the 90th percentile still represents about twice the mean momentum whatever the altitude. In contrast, the main difference lies in the contribution to the total flux of the $1 \%$ largest events, which seems to decrease in the HIRDLS observations in contrast with what was found in the numerical simulations. Several factors may contribute to this difference. Once again, the better sampling of the most infrequent events in the WRF simulations could be one reason. Besides, HIRDLS hardly resolves waves with horizontal wavelengths smaller than about $400 \mathrm{~km}$, which are, on the contrary, explicitly resolved in the numerical simulations. Finally, the vertical shear of the stratospheric zonal wind is larger in September/October than in November, which may result in an enhanced wave filtering in the former months, and therefore a decrease of the wave intermittency with height in the HIRDLS pdfs.

\section{Effect of filtering by the wind}

In this section, we study with the help of Monte Carlo simulations how the gravity wave filtering by the background flow is continuously modifying the shape of the momentum-flux pdfs as the waves propagate upward in the atmosphere. The following paragraphs will detail the approach that we used, which has been inspired in many respects by Hines (1993) and Souprayen et al. (2001). In 
particular, we have considered that the primary mechanism that shapes the pdfs is the progressive removal of gravity waves encountering their critical level, and have ignored in this first approach any others, such as the wave saturation induced by the exponential decrease of atmospheric density. Previous studies (Souprayen et al. 2001; Hertzog et al. 2002a) have already shown that this approach (which is used here in relation with momentumflux pdfs) is capable of generating the commonly observed $m^{-3}$ slope of the gravity wave energy spectrum at large $m$.

\section{a. Assumptions and theoretical framework}

The Monte Carlo simulations are thus aimed at describing the propagation of a set of gravity wave packets in a varying background atmosphere that spreads the initial wave spectrum and eventually causes the occurrence of critical levels for some of the waves. In the following, we will neglect the nonlinear wave-wave interactions that Hines (1991) considered as the primary cause for the Doppler spreading of the wave spectrum; that is, the modification of a given wave packet's wavenumbers by the resulting random wind fluctuations caused by the whole set of waves. Rather, we will follow the approach suggested in Souprayen et al. (2001), and consider that the wind fluctuations encountered along the wave packet trajectories are only caused by the large-scale flow. In this case, the random character of the wind fluctuations (which is needed to spread the wave spectrum and eventually cause the wave filtering) results from the fact that the waves observed on a given location in the stratosphere have propagated there from various places in the troposphere, and have thus probed various wind fields along their trajectories. This effect is likely even more significant for our own interest in the momentum-flux pdfs, since these pdfs are constructed by taking into account all the observations made in a wide geographical area (e.g., the southern polar cap) and for a long time period (at least 1 month). The pdfs therefore account for a large number of wave packets that have each propagated through different tropospheric weather patterns, and the resulting effect can thus be taken into account by considering the background wind as a random variate.

For simplicity, the Monte Carlo simulations are performed in the midfrequency approximation, that is,

$$
f \ll \hat{\omega} \ll N,
$$

with $f$ and $N$ the inertial and Brunt-Väsäilä frequencies, respectively. In this context, the dispersion relation for gravity waves reads

$$
m^{2}=\frac{N^{2}}{\hat{\omega}^{2}}\left(k^{2}+l^{2}\right)
$$

where $(k, l, m)$ are the zonal, meridional, and vertical components of the wave vector $\mathbf{k}$. Further assumptions are made for the background atmosphere: we first consider $N$ constant and make use of a typical stratospheric value, that is, $2 \times 10^{-2} \mathrm{rad} \mathrm{s}^{-1}$. The horizontal background flow $\mathbf{u}_{h}(s)$ (with $s$ an abscissa along the wave path $^{1}$ ) is then assumed to be time independent and horizontally homogeneous at the scales of gravity wave packets. In this context, the absolute frequency $\omega=$ $\hat{\omega}+\mathbf{k} \cdot \mathbf{u}_{h}$ of a given wave packet, as well as the horizontal components $(k, l)$ of the wave vector, remain constant during the wave propagation in the atmosphere. In contrast, $\hat{\omega}$ and $m$ are changing due to the variations of the background wind.

As we are only interested in upward-propagating waves, $\hat{\omega}$ and $m$ must have opposite signs, so that the dispersion relation can be written as

$$
m=-\frac{N}{\hat{\omega}} k_{h}
$$

where $k_{h}=\sqrt{k^{2}+l^{2}}$ is the horizontal wavenumber. We furthermore adopt the convention that $m<0$ until the wave packet encounters a critical level (where $m$ goes to infinity). Last, we consider for simplicity that the background wind is zero at the source level $s_{0}$ as follows:

$$
u_{h}\left(s_{0}\right) \equiv\left|\mathbf{u}_{h}\left(s_{0}\right)\right|=0 .
$$

The change of the wave packet vertical wavenumber $m_{0}$ at the source level to $m_{1}$ at a nearby upper level $s_{1}$ is then inferred from the dispersion and Doppler-shift relations as shown:

$$
m_{1}^{-1}=m_{0}^{-1}+\frac{u_{h}\left(s_{1}\right) \cos \theta\left(s_{1}\right)}{N}
$$

where $\theta\left(s_{1}\right)$ is the angle between the (constant) wave direction of propagation (hereafter referred to as $\varphi$ ) and the background wind at $s_{1}$. Hence, in the case where $\cos \theta\left(s_{1}\right)>0$, the wave packets for which $\left|m_{0}\right|$ is greater than a critical vertical wavenumber $m_{\varphi}^{c}\left(s_{1}\right)$, defined as

$$
m_{\varphi}^{c}\left(s_{1}\right) \equiv \frac{N}{u_{h}\left(s_{1}\right) \cos \theta\left(s_{1}\right)},
$$

\footnotetext{
${ }^{1}$ No assumption on how waves propagate in the atmosphere is needed at this stage.
} 
have encountered a critical level between $s_{0}$ and $s_{1}$. In our numerical simulations, we will simply assume that these wave packets are completely obliterated and that consequently the totality of their momentum flux is no longer in the wave field (but has been transferred to the mean flow).

\section{b. Monte Carlo simulations}

Unless otherwise stated, the Monte Carlo simulations of the momentum-flux pdfs proceed by starting from a power-law spectral distribution of momentum flux versus vertical wavenumber at the source level, that is, $\overline{u_{\|}^{\prime} w^{\prime}}\left(m_{0}\right) d m_{0} \sim m_{0}^{\beta} d m_{0}$. The figures shown in the next sections have thus been obtained with an initially flat $(\beta=0)$ momentum-flux spectrum, which corresponds to a kinetic energy spectrum $\overline{u_{\|}^{\prime}}\left(m_{0}\right)$ that scales as $m_{0}$ at low wavenumbers, as commonly reported in the atmosphere (e.g., Fritts and VanZandt 1993). Simulations with other momentum-flux source spectra have nevertheless been performed (with spectral laws varying from $\beta=-1$ to $\beta=1$ ), and provide essentially the same results. At the source level, this momentum flux is distributed within a range of vertical wavenumbers (from $\left|m_{\text {inf }}\right|$ to $\left.\left|m_{\text {sup }}\right|\right)$, and the initial total momentum flux is normalized in each numerical realization as shown:

$\rho\left(s_{0}\right) \int_{0}^{2 \pi} \int_{\left|m_{\text {inf }}\right|}^{\left|m_{\text {sup }}\right|} \overline{u_{\|\|^{\prime}}^{\prime}}\left(m_{0}\right) \Phi(\varphi) d m_{0} d \varphi=1$

where the function $\Phi(\varphi)$ describes the angular dependence in the horizontal plane of the source flux. This uniform normalization is intended to represent a wave source that emits gravity waves with always the same momentum flux, that is, a perfectly nonintermittent source. The evolution of the momentum-flux distribution during the wave propagation is thus entirely due to the filtering of part of the initial spectrum by the background wind.

At each propagation step, the critical vertical wavenumber is computed according to Eq. (9), and the momentum flux associated with that part of the source spectrum that has been filtered since the previous level is set to 0 . The remaining part is conservatively propagated to the next level. The remaining flux after $i$ propagation steps is thus

$F\left(s_{i}\right)=\rho\left(s_{0}\right) \int_{0}^{2 \pi} \int_{\left|m_{\mathrm{int} \mid}\right| m_{i, \varphi}^{c}}^{u_{\|}^{\prime} \boldsymbol{w}^{\prime}}\left(m_{0}\right) \Phi(\varphi) d m_{0} d \varphi$,

where

$$
m_{i, \varphi}^{c} \equiv \min _{j=0 \ldots i}\left[m_{\varphi}^{c}\left(s_{j}\right)\right]
$$

is the minimum critical wavenumber between the source level and the $i$ th step of propagation for waves propagating with an angle $\varphi$ with the zonal direction. [Equation (11) is only valid as long as the whole initial spectrum has not be filtered, that is, as long as $\left.m_{i, \varphi}^{c}>\left|m_{\text {inf }}\right|\right]$. Hence, $m_{i, \varphi}^{c}$ (through its dependence on the background wind) exclusively carries the random character of the momentum flux in the simulations.

Two different settings for the background wind have been used in the Monte Carlo simulations. The momentumflux pdfs resulting from these two settings are described in the following sections.

\section{c. Simulation with a random wind field}

In the first setting, designed to be as simple as possible, the background wind is assumed to be unidirectional (e.g., zonal) and is generated with a discrete Gaussian whitenoise process: a set of independent and identically distributed normal random variables with zero mean and uniform variance $\sigma_{u}^{2}$. This mimics the approach used by Hines (1993). ${ }^{2}$ By symmetry, we can then use a Dirac distribution for the angular dependence $\Phi(\varphi)=\delta(\varphi=\pi)$; that is, the waves propagate along only one direction of propagation, colinear to the wind direction (e.g., westward). We have used a standard deviation of $\sigma_{u}=7 \mathrm{~m} \mathrm{~s}^{-1}$, and the initial momentum flux is distributed between $\left|m_{\text {inf }}\right|=0.1$ cycles per kilometer and $\left|m_{\text {sup }}\right|=1$ cycles per kilometer, which corresponds to phase speeds with respect to the ground $\left(c_{h}=N / m\right)$ between 3 and $30 \mathrm{~m} \mathrm{~s}^{-1}$. The probability of filtering the whole wave field $\left(u_{h}>30 \mathrm{~m} \mathrm{~s}^{-1}\right)$ is thus marginal, as well as the probability of completely free propagation $\left(u_{h}<3 \mathrm{~m} \mathrm{~s}^{-1}\right)$. The following results are based on $10^{5}$ realizations of the background wind.

Figure 8 displays the evolution of the gravity wave momentum-flux pdfs in this random wind field. The initial single-value momentum flux is rapidly eroded due to the efficient filtering by the background wind, and a broad distribution quickly emerges. Most interestingly, the resulting pdfs are consistent with the lognormal distribution that appears in some observed pdfs after about 10 propagation steps. Sensitivity tests have been performed to assess how a change of the background wind standard deviation modifies these results. As expected, a larger $\sigma_{u}$ produces distributions with smaller fluxes, while a smaller $\sigma_{u}$ produces distributions with larger fluxes. The general agreement with the lognormal

\footnotetext{
${ }^{2}$ Note, in particular, that as in Hines (1993), the distance between two subsequent abscissas $s_{i}$ and $s_{i+1}$, which corresponds to the "decorrelation distance" of the background wind along the wave path, is left unspecified here. It would likely depend on the wave tridimensionnal direction of propagation and on the local atmospheric properties.
} 


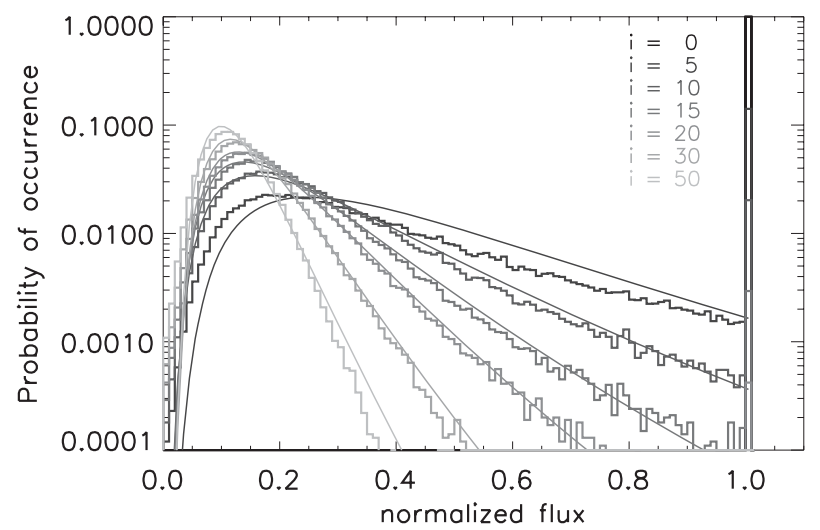

FIG. 8. Pdfs of gravity wave total momentum fluxes in a random Gaussian zonal wind field (with $\sigma_{u}=7 \mathrm{~m} \mathrm{~s}^{-1}$ ). The figure shows the pdfs after different propagation steps (as indicated in the topright corner). At the source level $(i=0)$, the (nondimensional) momentum flux is constant and equal to 1 . The continuous lines show the lognormal pdfs with the same geometric mean and standard deviation as the simulated pdfs for $i>0$. See text for details.

pdf is nevertheless conserved, provided that the range of values reached by the background wind is comparable to that of the wave phase speeds or, in other words, that the probability that part of the wave field is filtered at critical levels is high. We also studied the impact of introducing correlation in the background wind (e.g., simulating it using an autoregressive process rather than a decorrelated white-noise process): typically, the evolution of the pdf is slowed down, but the principal results remain valid.

The emergence of the lognormal distribution in these simulations can be understood as follows. The momentum flux $F\left(s_{n}\right)$ at abscissa $s_{n}$ along the wave track in the atmosphere is actually proportional to that at the previous abscissa as shown:

$$
F\left(s_{n}\right)=\alpha_{n} F\left(s_{n-1}\right)
$$

where $\alpha_{n}$ is a random number between 0 and 1 , which represents the contribution to $F\left(s_{n-1}\right)$ of that part of the wave field that has propagated conservatively between $s_{n-1}$ and $s_{n}$. Hence, linking $F\left(s_{n}\right)$ to the constant value of the flux at the source level, one gets

$$
F\left(s_{n}\right)=\prod_{i=1}^{n} \alpha_{i} F\left(s_{0}\right)
$$

Such a multiplicative process is known to yield a lognormal random variate [which can be easily seen by taking the log of Eq. (14) and invoking the central limit theorem], as long as the $\alpha_{i}$ s are independent and well behaved (Aitchison and Brown 1957). This last condition is, in fact, linked to the background wind range being similar to that of the wave phase speeds, as already mentioned.

\section{d. Simulation with a random wind field and a stochastic source}

We study here how the momentum-flux pdfs are modified if one uses a finite number nw of discrete wave packets to represent the wave source instead of a full continuous spectrum as in the previous subsection. Indeed, as mentioned in the introduction, several GWD parameterizations are based on the use of discrete wave packets. Recent developments (Eckermann 2011; Lott et al. 2012) have furthermore demonstrated that these parameterizations produce the same large-scale atmospheric response when only a few wave packets with randomly chosen characteristics (e.g., phase speeds, wavenumbers) are used per model grid point, instead of several tens with determined characteristics. These new parameterizations are termed stochastic as the random wave packets change from one grid point and time step to the other, so that the full initial phase speed or wavenumber distribution of gravity wave flux is only recovered after averaging in model space and/or time.

The source spectrum in our simulation is thus discretized with $\mathrm{nw}=1,10$, or 100 wave packets, with vertical wavenumbers chosen randomly between $m_{\text {inf }}$ and $m_{\text {sup }}$ with equal probability. The flux carried by each packet is normalized, so that

$$
\sum_{j=1}^{\mathrm{nw}} u_{\|\|^{\prime}} \boldsymbol{w}^{\prime}\left(m_{0_{j}}\right)=1
$$

where the wave packets are assumed to have a Dirac distribution in the $m$ space. In this expression, $m_{0_{j}}$ is the vertical wavenumber of packet $j$ at the source level. The stochastic source simulations have been done with the same underlying $m^{-1}, m^{0}$, and $m$ dependence of the source flux spectrum, and the results were found independent of this choice. We once again illustrate the results with the $m^{0}$ source distribution. In this case, the initial flux of each wave packet is thus simply $1 / n w$. [We note in passing that the flux carried by each wave packet is not randomly chosen, but known once the wave packet vertical wavenumber is determined, in agreement with Eckermann (2011) stochastic parameterization.] The nw wave packets are then launched in one realization of the background wind, with the same mean and standard deviation as in the previous section. The resulting pdfs are constructed by performing $10^{4}$ different background wind realizations.

The momentum-flux pdfs obtained with the stochastic source are compared to those obtained previously with 

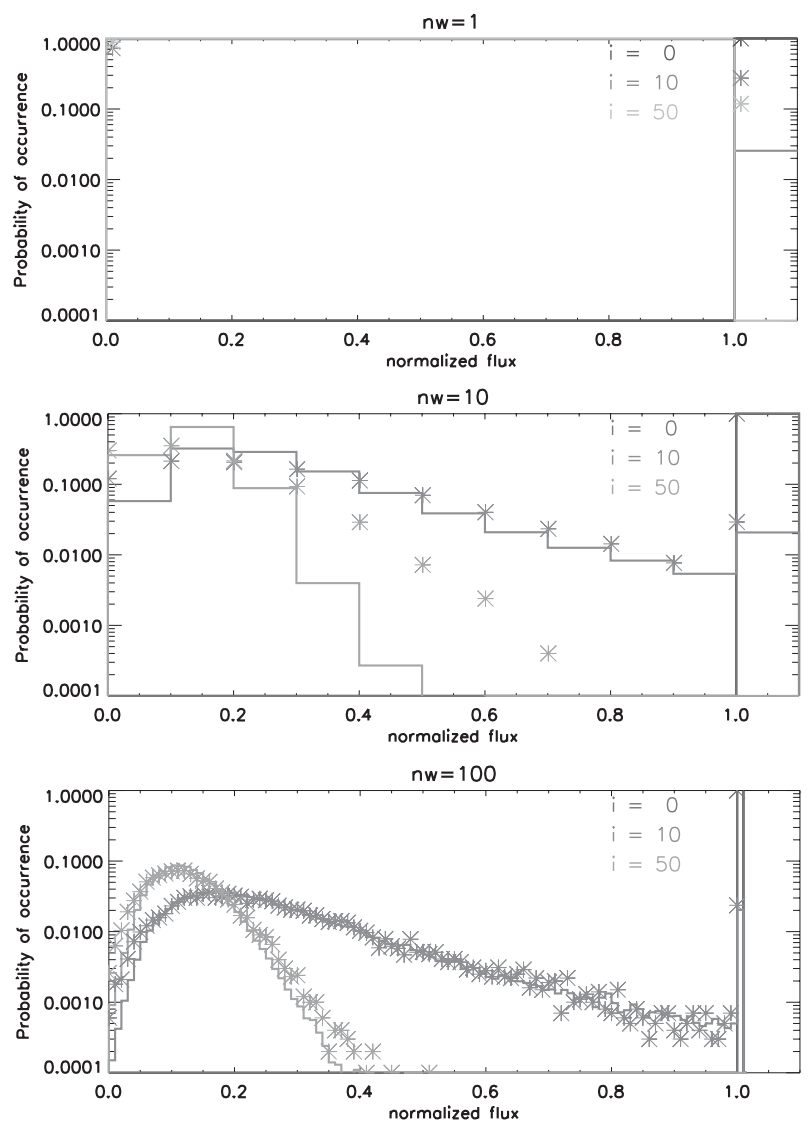

FIG. 9. Pdfs of gravity wave total momentum fluxes in the random Gaussian zonal wind field simulation. The panels show the pdfs after different vertical propagation steps (as indicated in the top-right corner of each panel). The pdfs produced by the "stochastic source" are displayed with asterisks. The pdfs were obtained by launching either (top) $\mathrm{nw}=1$, (middle) $\mathrm{nw}=10$, or (bottom) nw $=100$ wave packets per background wind realization. The pdf obtained with the full launch spectrum (i.e., as in Fig. 8) is displayed with continuous lines. The resolution of this pdf is adapted to the number of wave packets used in the stochastic source simulation (see text for details).

the full launch spectrum in Fig. 9. The resolution of the latter pdf is, however, degraded with respect to that shown in Fig. 8. Let us actually consider the case with a single wave packet per wind realization $(\mathrm{nw}=1)$. The single wave packet carries the whole momentum flux, and at propagation step $i$, the momentum flux can therefore only take two values: 1 if the wave packet has succeeded to propagate to this step from the source or 0 if it has been filtered in between. Turning back to the general case, the momentum-flux pdfs can take nw +1 values in the stochastic source simulations. The full launch spectrum pdfs are accordingly shown in Fig. 9 with bin widths equal to $1 / \mathrm{nw}$. Not surpringly, the nw $=1$ simulation is unable to reproduce the characteristics of the momentum-flux distribution obtained with the full

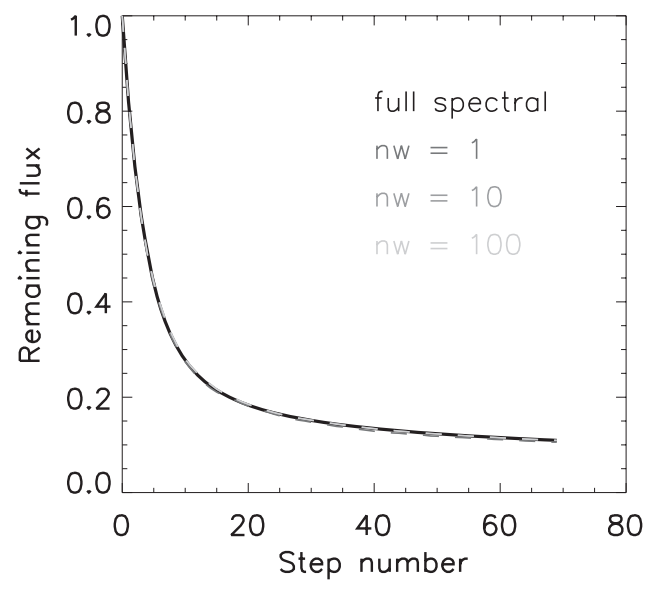

FIG. 10. Mean momentum flux remaining after the number of propagation steps shown in the abscissa in the random wind simulation. The flux associated with the full launch spectrum source is represented with a continuous line. Those corresponding to the stochastic source are represented with dashed lines, and the gray scale indicates the number of wave packets used.

launch spectrum. However, using 100 wave packets produces an excellent agreement with the spectral source. One may also argue that the $\mathrm{nw}=10$ simulation starts to retain essential features of the momentum-flux pdf (e.g., the broad tail), and might therefore provide a good tradeoff between computational cost and momentum-flux intermittency in operational GWD parameterizations.

The ability of the stochastic source to generate momentum-flux pdfs that more or less agree with those obtained with the full spectral source tells, however, nothing on how well the mean momentum flux, averaged over all the random wind realizations, corresponds to that obtained with the full spectral source. Figure 10 actually shows that, whatever the number nw of wave packets in the stochastic source, the mean momentum flux produced by the stochastic source is almost indistinguishable from that of the full spectral source. This result mirrors the findings reported in Eckermann (2011), who succeeded in obtaining a realistic mean-flow forcing by running a stochastic GWD parameterization with a single-wave packet source. In our simulations, this result likely arises from both the large number of realizations and the properties of the wave-filtering process, which is constrained by the background wind profile that has constant statistical characteristics. In GCM runs, the large number of model grid points and the ergodic character of the large-scale circulation may provide companion agreements.

\section{e. Simulation with a realistic wind field}

To assess how the previous approach of the wave pdf generation may work with more a realistic wind field, 


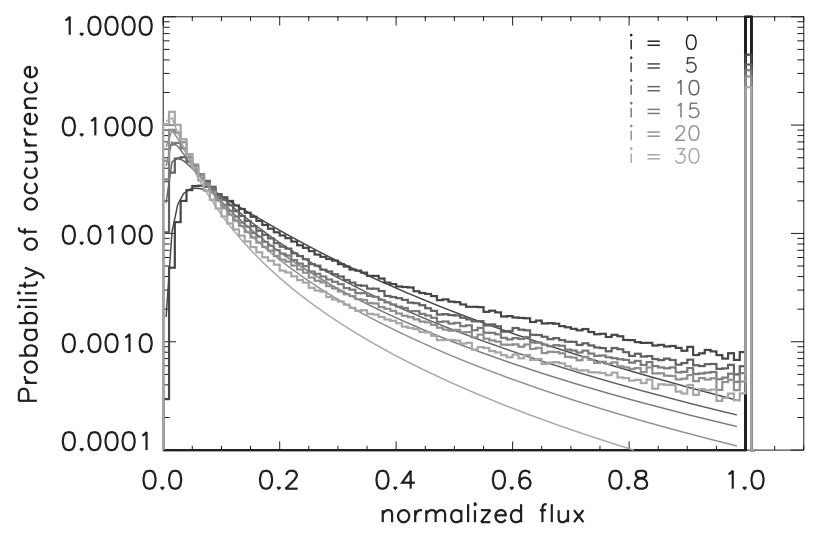

FIG. 11. As in Fig. 8, but for the simulations with the ECMWF wind field.

another set of simulations has been performed with the 0000 UTC analyzed winds provided by the ECMWF Integrated Forecast System. In this case, the analyses for October 2010 south of $50^{\circ} \mathrm{S}$ have been used, so as to benefit from the highest possible resolution of the model: $0.125^{\circ} \times 0.125^{\circ}$ in the horizontal, 44 levels from about $700 \mathrm{hPa}$ (arbitrarily chosen as the source level) to $40 \mathrm{hPa}$ in the vertical. The resulting pdfs are thus obtained from about $9 \times 10^{5}$ individual simulations.

In the previous simulations with the random wind field, we made the assumption that the wind values were those felt by the waves during their propagation. Such an approach could be reproduced with a realistic wind field by performing ray-tracing simulations. However, to reduce the computation cost and to mimic what is currently done with GWD parameterizations, we simply assumed here that the waves propagate vertically in the atmosphere. In these ECMWF simulations, we furthermore used an isotropic wave momentum flux at the source, that is, $\Phi(\varphi)=1 / 2 \pi$. This isotropic source is implemented by picking a single direction of propagation in a uniform distribution between 0 and $2 \pi$ at each ECMWF grid point. We otherwise used the same full launch spectrum as in the random wind simulations, except that we extended the range of the source wavenumbers by using $\left|m_{\text {inf }}\right|=0.05$ cycles per kilometer and $\left|m_{\text {sup }}\right|=10$ cycles per kilometer, corresponding to phase speeds between 0.3 and $60 \mathrm{~m} \mathrm{~s}^{-1}$.

The resulting pdfs are displayed in Fig. 11. As in the random wind simulations, a broad momentum-flux pdf with a shape that shares many similarities with the observed ones is rapidly produced. Two features are nonetheless different: first, despite the extended range of ground-based phase speeds, the whole wave field is able to propagate freely up to $40 \mathrm{hPa}$ in a significant number of individual simulations, as highlighted by the pdf peak at $F\left(s_{i}\right)=1$. These simulations essentially correspond to waves propagating toward the west in the mainly eastward midlatitude October flow of the Southern Hemisphere. Second, the agreement between the produced pdfs and the lognormal distribution is only obtained for the smallest momentum-flux values (up to $0.2-0.4$ ). This last feature is also reminiscent of the observed pdfs above mountainous areas in September/ October.

These two differences are associated with the fact that the ECMWF wind field is, from the point of view of wave filtering, less random than the one used in the first simulation. In particular, the mainly eastward flow in the upper troposphere and the stratosphere implies that $\alpha_{i}=1$ in Eq. (14) from a certain level onward for the westward-propagating waves, so that the conditions for the emergence of the lognormal distribution are no longer met.

Simulations with a stochastic source (instead of the full launch spectrum) in the ECMWF winds have been performed too, and the results were found very similar to those reported in the previous section: the pdf shape displayed in Fig. 11 emerges with a relatively low number of wave packets (i.e., nw $=10$ ), while the mean momentum flux does not depend on nw.

\section{Summary and concluding remarks}

The first part of this article was aimed at studying the properties of gravity wave momentum-flux pdfs in the upper troposphere and lower stratosphere over Antarctica and the Southern Ocean. The pdfs have been obtained independently from in situ balloonborne measurements, limb-sounding observations of the atmosphere, and high-resolution numerical simulations. In all these data sources, the momentum-flux pdfs exhibit long tails that span over (at least) one or two orders of magnitude, and therefore highlight the so-called intermittency of gravity wave activity in the lower stratosphere. It has been, for instance, shown that for the region considered in this study, about $60 \%$ of the total observed flux is only due to the $10 \%$ largest fluxes when the winter regime of the stratospheric circulation is established (and a large fraction of the waves can propagate almost freely to the stratosphere). This figure is even more pronounced above mountainous areas, where typically $25 \%$ of the total flux is only produced by $1 \%$ of the events. We have also found that these characteristics of the atmospheric gravity wave field keep essentially constant with altitude, as long as the vertical shear of the horizontal wind remains eastward in the stratosphere. In contrast, the intermittency of gravity wave activity decreases significantly during the transition from winter to summer circulation. While these 
features are found to be fairly robust whatever the dataset used, the characteristics of the momentum-flux pdfs likely deserve to be further confirmed in other places and seasons than those studied here. Using the pdfs to assess the overall contribution of momentum fluxes beyond certain percentiles as was done here [or to compute a more synthetic metric, such as the Gini coefficient proposed by Plougonven et al. (2012)] actually provides a useful way to quantify gravity wave intermittency.

The second part of this article described the role played by wave filtering at critical levels in modifying the shape of momentum-flux pdfs. Simple Monte Carlo simulations have been designed to study the propagation of gravity waves through a varying background wind field. In the simulations with a random background wind with values similar to those of the wave phase speeds, the numerous occurrence of critical levels that filter out a portion of the initial wave spectrum tends to modify the single-value pdf used at the source level (corresponding to a constant, homogeneous source) toward lognormal pdfs, which are actually observed either above oceanic areas during winter or summer. As first suggested by Hines (1993), such evolution can be understood as the multiplicative result of discrete filtering events along the wave propagation, each event corresponding to the deposition of a fraction of the remaining flux. In contrast, when the wind field is such that a large portion of the gravity wave field can propagate almost freely (as was the case in the simulations with the November ECMWF-analyzed winds), significant departures from the lognormal distribution are observed. These departures are typically encountered in the observations above mountainous areas during winter (in which case, the sporadic wave emission may also contribute to the observed intermittency in the lower stratosphere). These results were found to be rather independent of how the wave source was treated, either as a full spectrum or as a superposition of $\geqslant 10$ independent wave packets. Finally, the limitations of our simulations should be stressed: in particular, neither the wave-induced fluctuations of the background wind nor the saturation of individual wave packets were taken into account. Both processes can enhance the deposition of gravity wave momentum flux and therefore modify the momentum-flux pdfs.

The sporadic gravity wave activity exemplified in this article can provide some observational grounds for the development and use of novel stochastic gravity wave drag parameterizations in general circulation models (e.g., Piani et al. 2004; Eckermann 2011; Lott et al. 2012). But it may also be worthwhile to assess how the observed gravity wave intermittency is simulated by current deterministic GWD parameterizations. On the one hand, one could expect that these parameterizations simulate, at least to some extent, the filtering of the gravity wave field by the background wind, which, as we suggested, is an important factor in producing the observed pdfs. On the other hand, most nonorographic GWD parameterizations use a constant and/or homogeneous wave source, and therefore likely underestimate the real wave intermittency. Such study will be the subject of future work.

Acknowledgments. The authors would like to acknowledge CNES for the long-standing development of stratospheric superpressure balloons and for the successful Vorcore balloon campaign in 2005. The study benefited from stimulating discussions at the International Space Science Institute (ISSI) during the gravity wave group meetings. We would like in particular to thank Julio Bacmeister, Adam Scaife, and Robert A. Vincent for their ideas and suggestions. This work was initiated during Joan Alexander's visit at LMD, which was funded by École Normale Supérieure. The European Centre for MediumRange Weather Forecasts provided the meteorological analyses used in this study. The WRF simulations were performed on HPC resources from GENCI-IDRIS (Grants 2009 and 2010-012039). Work by MJA was supported by the NASA Atmospheric Chemistry-Aura Science Team Program, Contract NNH11CD32C. The authors would also like to thank two anonymous reviewers for their insightful remarks: the stochastic source experiments were indeed performed according to one reviewer's suggestion.

\section{REFERENCES}

Aitchison, J., and J. A. C. Brown, 1957: The Lognormal Distribution, with Special Reference to Its Use in Economics. Cambridge University Press, $176 \mathrm{pp}$.

Alexander, M. J., and L. Pfister, 1995: Gravity wave momentum flux in the lower stratosphere over convection. Geophys. Res. Lett., 22, 2029-2032.

—_, and T. J. Dunkerton, 1999: A spectral parameterization of mean-flow forcing due to breaking gravity waves. J. Atmos. Sci., 56, 4167-4182.

— amplitude mountain wave event over the Antarctic peninsula. J. Geophys. Res., 112, D21103, doi:10.1029/2006JD008368.

— , and Coauthors, 2008: Global estimates of gravity wave momentum flux from High Resolution Dynamics Limb Sounder observations. J. Geophys. Res., 113, D15S18, doi:10.1029/ 2007JD008807.

— S. D. Eckermann, D. Broutman, and J. Ma, 2009: Momentum flux estimates for South Georgia Island mountain waves in the stratosphere observed via satellite. Geophys. Res. Lett., 36, L12816, doi:10.1029/2009GL038587.

_ , and Coauthors, 2010: Recent developments in gravity-wave effects in climate models and the global distribution of 
gravity-wave momentum flux from observations and models. Quart. J. Roy. Meteor. Soc., 136, 1103-1124.

Andrews, D. G., J. R. Holton, and C. B. Leovy, 1987: Middle Atmosphere Dynamics. Academic Press, 490 pp.

Baumgaertner, A. J. G., and A. J. McDonald, 2007: A gravity wave climatology for Antarctica compiled from Challenging Minisatellite Payload/Global Positioning System (CHAMP/GPS) radio occultations. J. Geophys. Res., 112, D05103, doi:10.1029/ 2006JD007504.

Boccara, G., A. Hertzog, R. A. Vincent, and F. Vial, 2008: Estimation of gravity wave momentum flux and phase speeds from quasi-Lagrangian stratospheric balloon flights. Part I: Theory and simulations. J. Atmos. Sci., 65, 3042-3055.

Bühler, O., 2003: Equatorward propagation of intertia-gravity waves due to steady and intermittent wave sources. J. Atmos. Sci., 60, 1410-1419.

Dewan, E. M., 1997: Saturated-cascade similitude theory of gravity wave spectra. J. Geophys. Res., 102 (D25), 29 799-29 817.

_ - and R. E. Good, 1986: Saturation and the "universal" spectrum for vertical profiles of horizontal scalar winds in the atmosphere. J. Geophys. Res., 91 (D2), 2742-2748.

Eckermann, S. D., 2011: Explicitly stochastic parameterization of nonorographic gravity wave drag. J. Atmos. Sci., 68, 1749-1765.

_ , and P. Preusse, 1999: Global measurements of stratospheric mountain waves from space. Science, 286, 1534-1537.

Fritts, D. C., and W. Lu, 1993: Spectral estimates of gravity wave energy and momentum fluxes. Part II: Parameterization of wave forcing and variability. J. Atmos. Sci., 50, 3695-3713.

_ - and T. E. VanZandt, 1993: Spectral estimates of gravity wave energy and momentum fluxes. Part I: Energy dissipation, acceleration, and constraints. J. Atmos. Sci., 50, 3685-3694.

_, T. Tsuda, S. Kato, T. Sato, and S. Fukao, 1988: Observational evidence of a saturated gravity wave spectrum in the troposphere and lower stratosphere. J. Atmos. Sci., 45, 17411759.

Gille, J., and Coauthors, 2008: High Resolution Dynamics Limb Sounder: Experiment overview, recovery, and validation of initial temperature data. J. Geophys. Res., 113, D16S43, doi:10.1029/ 2007JD008824.

Hertzog, A., and F. Vial, 2001: A study of the dynamics of the equatorial lower stratosphere by use of ultra-long-duration balloons 2. Gravity waves. J. Geophys. Res., 106 (D19), $22745-22761$

_ C. Souprayen, and A. Hauchecorne, 2002a: Eikonal simulations for the formation and the maintenance of atmospheric gravity wave spectra. J. Geophys. Res., 107, 4145, doi:10.1029/ 2001JD000815.

- , F. Vial, C. R. Mechoso, C. Basdevant, and P. Cocquerez, 2002b: Quasi-Lagrangian measurements in the lower stratosphere reveal an energy peak associated with near-inertial waves. Geophys. Res. Lett., 29, 1229, doi:10.1029/ 2001 GL014083.

—_, and Coauthors, 2007: Stratéole/Vorcore-Long-duration, superpressure balloons to study the Antarctic lower stratosphere during the 2005 winter. J. Atmos. Oceanic Technol., 24, 2048-2061.

—, G. Boccara, R. A. Vincent, and F. Vial, 2008: Estimation of gravity wave momentum flux and phase speeds from quasiLagrangian stratospheric balloon flights. Part II: Results from the Vorcore campaign in Antarctica. J. Atmos. Sci., 65, 3056-3070.

Hines, C. O., 1991: The saturation of gravity waves in the middle atmosphere. Part II: Development of Doppler-spread theory. J. Atmos. Sci., 48, 1360-1379.
1993: The saturation of gravity waves in the middle atmosphere. Part IV: Cutoff of the incident wave spectrum. J. Atmos. Sci., 50, 3045-3060.

_ 1997a: Doppler-spread parameterization of gravity-wave momentum deposition in the middle atmosphere. Part 1: Basic formulation. J. Atmos. Sol.-Terr. Phys., 59, 371-386.

— 1997b: Doppler-spread parameterization of gravity-wave momentum deposition in the middle atmosphere. Part 2: Broad and quasi monochromatic spectra, and implementation. J. Atmos. Sol.-Terr. Phys., 59, 387-400.

Holton, J. R., 1983: The influence of gravity wave breaking on the general circulation of the middle atmosphere. J. Atmos. Sci., 40, 2497-2507.

— , P. H. Haynes, M. E. McIntyre, A. R. Douglass, R. B. Hood, and L. Pfister, 1995: Stratosphere-troposphere exchange. Rev. Geophys., 33, 405-439.

Lindzen, R. S., 1981: Turbulence and stress owing to gravity wave and tidal breakdown. J. Geophys. Res., 86 (C10), 9707-9714.

Lorenz, M. O., 1905: Methods of measuring the concentration of wealth. Publ. Amer. Stat. Assoc., 9, 209-219.

Lott, F., L. Guez, and P. Maury, 2012: A stochastic parameterization of non-orographic gravity waves: Formalism and impact on the equatorial stratosphere. Geophys. Res. Lett., 39, L06807, doi:10.1029/2012GL051001.

Medvedev, A. S., and G. P. Klaassen, 1995: Vertical evolution of gravity wave spectra and the parameterization of associated wave drag. J. Geophys. Res., 100 (D12), 25 841-25 853.

Morgenstern, O., and Coauthors, 2010: Review of the formulation of present-generation stratospheric chemistry-climate models and associated external forcings. J. Geophys. Res., 115, D00M02, doi:10.1029/2009JD013728.

Nastrom, G. D., and K. S. Gage, 1985: A climatology of atmospheric wavenumber spectra of wind and temperature observed by commercial aircraft. J. Atmos. Sci., 42, 950-960.

— waves. Part I: Topographic excitation. J. Atmos. Sci., 49, 101-110.

Pfister, L., and Coauthors, 1993: Gravity waves generated by a tropical cyclone during the STEP tropical field program: A case study. J. Geophys. Res., 98 (D5), 8611-8638.

Piani, C., W. A. Norton, and D. A. Stainforth, 2004: Equatorial stratospheric response to variations in deterministic and stochastic gravity wave parameterizations. J. Geophys. Res., 109, D14101, doi:10.1029/2004JD004656.

Plougonven, R., A. Hertzog, and H. Teitelbaum, 2008: Observations and simulations of a large amplitude mountain wave breaking over the Antarctic peninsula. J. Geophys. Res., 113, D16113, doi:10.1029/2007JD009739.

_ _ A. Arsac, A. Hertzog, L. Guez, and F. Vial, 2010: Sensitivity study for mesoscale simulations of gravity waves above Antarctica during Vorcore. Quart. J. Roy. Meteor. Soc., 136, 1371-1377.

, A. Hertzog, and L. Guez, 2012: Gravity wave over Antarctica and the Southern Ocean: Consistent momentum fluxes in mesoscale simulations and stratospheric balloon observations. Quart. J. Roy. Meteor. Soc., doi:10.1002/qj.1965, in press.

Scinocca, J. F., 2003: An accurate spectral nonorographic gravity wave drag parameterization for general circulation models. J. Atmos. Sci., 60, 667-682.

Sidi, C., J. Lefrère, F. Dalaudier, and J. Barat, 1988: An improved atmospheric buoyancy waves spectrum model. J. Geophys. Res., 93 (D1), 774-790.

Skamarock, W., and Coauthors, 2008: A description of the Advanced Research WRF version 3. NCAR Tech. Note. NCAR/ TN-475+STR, 113 pp 
Smith, R. B., 1979: The influence of mountains on the atmosphere. Advances in Geophysics, Vol. 21, Academic Press, 87-230.

Smith, S. A., D. C. Fritts, and T. E. VanZandt, 1987: Evidence for a saturated spectrum of atmospheric gravity waves. J. Atmos. Sci., 44, 1404-1410.

Souprayen, C., J. Vanneste, A. Hertzog, and A. Hauchecorne, 2001: Atmospheric gravity wave spectra: A stochastic approach. J. Geophys. Res., 106 (D20), 24 071-24 086.

VanZandt, T. E., 1982: A universal spectrum of buoyancy waves in the atmosphere. Geophys. Res. Lett., 9, 575-578.

— , and D. C. Fritts, 1989: A theory of enhanced saturation of the gravity wave spectrum due to increases in atmospheric stability. Pure Appl. Geophys., 130, 399-420.

Vial, F., A. Hertzog, C. R. Mechoso, C. Basdevant, P. Cocquerez, V. Dubourg, and F. Nouel, 2001: A study of the dynamics of the equatorial lower stratosphere by use of ultra-long-duration balloons 1. Planetary scales. J. Geophys. Res., 106 (D19), 22 725-22 743.
Vincent, R. A., A. Hertzog, G. Boccara, and F. Vial, 2007: QuasiLagrangian superpressure balloon measurements of gravitywave momentum fluxes in the polar stratosphere of both hemispheres. Geophys. Res. Lett., 34, L19804, doi:10.1029/ 2007GL031072.

Warner, C. D., and M. E. McIntyre, 2001: An ultrasimple spectral parameterization for nonorographic gravity waves. J. Atmos. Sci., 58, 1837-1857.

Watanabe, S., Y. Kawatani, Y. Tomikawa, K. Miyazaki, M. Takahashi, and K. Sato, 2008: General aspects of a T213L256 middle atmosphere general circulation model. J. Geophys. Res., 113, D12110, doi:10.1029/2008JD010026.

Weinstock, J., 1990: Saturated and unsaturated spectra of gravity waves and scale-dependent diffusion. J. Atmos. Sci., 47, 2211-2225.

Wu, D. L., 2004: Mesoscale gravity wave variances from AMSU-A radiances. Geophys. Res. Lett., 31, L12114, doi:10.1029/ 2004GL019562. 